\title{
Molecular targeting of liposomal nanoparticles to tumor microenvironment
}

\author{
This article was published in the following Dove Press journal: \\ International Journal of Nanomedicine \\ 27 December 2012 \\ Number of times this article has been viewed
}

\author{
Gang Zhao',2 \\ B Leticia Rodriguez ${ }^{2}$ \\ 'Institute of Materia Medica, Shandong \\ Academy of Medical Science, \\ Shandong, China; ${ }^{2}$ Pharmaceutics \\ Division, College of Pharmacy, \\ The University of Texas at Austin, \\ Austin, TX, USA
}

\begin{abstract}
Liposomes are biodegradable and can be used to deliver drugs at a much higher concentration in tumor tissues than in normal tissues. Both passive and active drug delivery by liposomal nanoparticles can significantly reduce the toxic side effects of anticancer drugs and enhance the therapeutic efficacy of the drugs delivered. Active liposomal targeting to tumors is achieved by recognizing specific tumor receptors through tumor-specific ligands or antibodies coupled onto the surface of the liposomes, or by stimulus-sensitive drug carriers such as acid-triggered release or enzyme-triggered drug release. Tumors are often composed of tumor cells and nontumor cells, which include endothelial cells, pericytes, fibroblasts, stromal, mesenchymal cells, innate, and adaptive immune cells. These nontumor cells thus form the tumor microenvironment, which could be targeted and modified so that it is unfavorable for tumor cells to grow. In this review, we briefly summarized articles that had taken advantage of liposomal nanoparticles as a carrier to deliver anticancer drugs to the tumor microenvironment, and how they overcame obstacles such as nonspecific uptake, interaction with components in blood, and toxicity. Special attention is devoted to the liposomal targeting of anticancer drugs to the endothelium of tumor neovasculature, tumor associated macrophages, fibroblasts, and pericytes within the tumor microenvironment.
\end{abstract}

Keywords: tumor microenvironment, endothelium, neovasculature, tumor-associated macrophages, cationic liposomes, ligand- or antibody-mediated targeting

\section{Introduction}

Cancer is a disease that is difficult to eradicate. According to the most recent statistics from the International Agency for Research on Cancer, about 12.7 million cancer cases and 7.6 million cancer deaths occurred in 2008 worldwide. ${ }^{1}$ In general, cytotoxic drugs kill tumor cells, but also frequently display unwanted toxicities as they lack tumor cell selectivity. Moreover, drug resistance is often developed. ${ }^{2,3}$ Drug resistance limits the efficacy of chemotherapy in cancer patients and is typically due to reduced accumulation of drugs in tumor cells and acquired tumor cell protection from apoptosis. The genetic and epigenetic heterogeneity of tumors in combination with the selection of anticancer drugs leads to the overgrowth of drug-resistant variants. ${ }^{4}$ The adaptive phenotypes of cancer cells can be explained in part by genetic and epigenetic instability and mutations. ${ }^{5} \mathrm{~A}$ fivefold increase in genetic instability was found in cells grown in vivo compared to in vitro, ${ }^{6}$ indicating that the tumor microenvironment induces genetic changes that are not observed in vitro. Human solid tumors are heterogeneous and contain both neoplastic and normal cells. ${ }^{7}$ The loss of apoptotic potential is most likely driven by the genomic instability and inhibition in deoxyribonucleic acid (DNA) repair induced by the tumor microenvironment. ${ }^{8}$ Hypoxia and reoxygenation often lead to the formation
Correspondence: Gang Zhao Institute of Materia Medica, Shandong Academy of Medical Science, 18877 Jingshi Road, Jinan, Shandong, 250062, China Tel +86053 I 829| 9973 Fax +86053182615996 Email zhaogangcnjn@yahoo.com.cn 
of reactive oxygen species that have been shown to induce damage in the DNA. Reactive oxygen species formation leads to the amplification of mutations, single and point mutations, as well as single and double strand breaks. ${ }^{5}$ Finally, tumor cells also have the ability to avoid regulatory control mechanisms, leading to subpopulations with an aggressive phenotype.

To circumvent such obstacles, attention has been focused on killing other nontumor cells present in the tumor microenvironment. There is an emerging effort to better explain and predict the phenotypic characteristic of cancer, and there is evidence suggesting that the tumor stromal tissue is not a passive bystander in tumor development. ${ }^{9}$ The tumor microenvironment includes cancer cells, stromal cells, immune cells, fibroblasts, cytokines, vascular tissue, and the extracellular matrix (Figure 1). ${ }^{10}$ Tumor cells interact with the surrounding nonneoplastic cells such as endothelial cells, cancer-associated fibroblasts, mesenchymal stem cells, and different immune cells such as lymphocytes and tumor-associated macrophages (TAMs). ${ }^{11}$ Unlike tumor cells, the supporting cells in tumor microenvironments are often genetically stable, which reduces the potential for the development of drug resistance.

Finally, the idea that tumors may contain cancer stem cells has reshaped approaches for tumor chemotherapy and targeted drug delivery. Cytotoxic chemotherapy has been mainly aimed at killing proliferating tumor cells, and most chemotherapeutic drugs kill tumor cells by interfering with cell division. However, cancer stem cells are mostly quiescent and resistant to apoptosis. Therefore, cancer stem cells may survive conventional chemotherapy and cause the repopulation of cancers or relapse, making it necessary to develop novel and effective approaches that can eradicate cancer stem cell populations as well. In fact, there have been attempts to utilize liposomes to address this need. For example, retinoic acids, derivatives of vitamin A, are known to induce cancer cell differentiation, proliferation arrest, and apoptosis. Li et al $^{12}$ incorporated all-trans retinoic acid into stealth liposomes and showed that combination therapy using retinoic acid-liposomes and vinorelbine liposomes was more effective than monotherapy using vinorelbine liposomes alone in inhibiting the relapse of breast cancer arisen from breast cancer stem cells, likely because the retinoic acidliposomes promoted the differentiation of cancer stem cells and arrested cell cycle. ${ }^{12}$

In this review, we will briefly summarize studies that took advantages of liposomal nanoparticles as a carrier to deliver anticancer drugs to the tumor microenvironment. Special attention is devoted to the liposomal targeting of anticancer drugs to the endothelium of tumor neovasculature, tumor associated macrophages, fibroblasts, and pericytes within the tumor microenvironment. Figure 1 also includes some

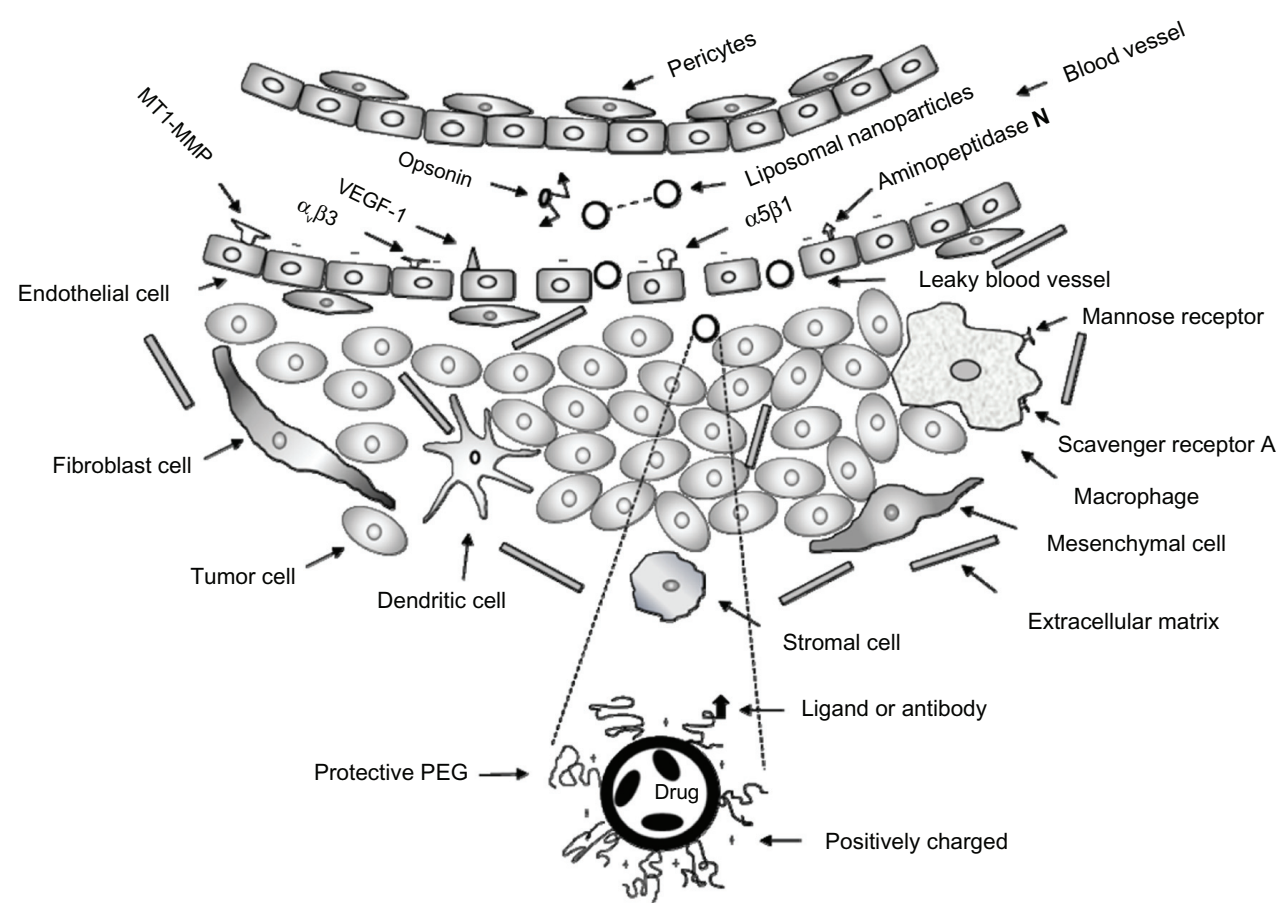

Figure I Depiction of tumor cell and nontumor cell microenvironment including potential targets used for liposomal delivery. Abbreviations: MTI-MMP, membrane type-I matrix metalloprotease; VEGF-I, vascular endothelial growth factor-I; PEG, polyethylene glycol. 
of the cells and molecules that had been used as targets for liposomes.

\section{Endothelial cells in tumor neovasculature}

Angiogenesis is an important process in tumor development as it provides soluble nutrients as well as oxygen through circulation. ${ }^{13-15}$ In normal quiescent vasculature, the tissue is comprised of endothelial cells, pericytes, and basement membrane. The endothelial tubule is maintained at a quiescent state with a layer of pericytes. ${ }^{16}$ In order to form new blood vessels, pericytes must be dissociated from the endothelial tubule, followed by basement membrane degradation. Extravascular fibrin deposition occurs, and finally endothelial cells must respond to proangiogenic signals and initiate the formation of new tubules. ${ }^{17}$ Activated endothelium of newly formed vessels differs from mature blood vessels based on the high expression of specific molecules. ${ }^{18}$ Although tumor blood vessels are heterogeneous, vascular endothelial growth factor (VEGF)-A serves as a critical factor, promoting new blood vessel formation in tumors. ${ }^{19}$ TAMs secrete VEGF, fibroblast growth factor (FGF)-2, platelet derived growth factor (PDGF), and epidermal growth factor (EGF). Together, these signaling molecules initiate the formation of new blood vessels from existing blood vessels. ${ }^{20}$

\section{Immune cells in tumor microenvironment}

Malignant tumors are rich in immune cells, such as macrophages, neutrophils, dendritic cells (DCs), and natural killer cells. ${ }^{21}$ Recent studies have shown that the accumulation of macrophages in tissues of chronic inflammation promotes cancer progression by stimulating tumor vascularization, invasion, and metastasis. ${ }^{22}$ Further, it was shown that TAMs correlated with poor prognosis for cancer patients. ${ }^{23}$ In certain mouse cancer models, CD4+ helper T lymphocytes and B lymphocytes release protumorigenic factors such as interleukin (IL)-4, IL-10, transforming growth factor beta, which stimulate the tumor-promoting properties of macrophages. ${ }^{24,25}$ TAMs are required for tumor cell migration, invasion, and metastatic formation. ${ }^{26}$ TAMs from differentiated monocytes are recruited to tumor sites in response to tumor chemotactic factors. Importantly, it was shown that women with tumors that have a combination of high TAMs, high CD4+ T helper cells, and low cytotoxic CD8+ T cells are at a high risk of developing secondary tumors. ${ }^{2}$ Moreover, TAMs are known to stimulate excessive tumor vascularization, and their persistent expression of proangiogenic factors, such as VEGF, induces the formation of abnormal hypoperfused blood vessels, which limit the delivery of chemotherapy to tumors. ${ }^{27,28}$

TAMs have also been shown to accumulate in hypoxic tumor areas. DCs are the most important antigen-presenting cells and can affect the functions of natural killer cells, which are effector cells of the innate immune system, ${ }^{29}$ and are capable of killing virus-infected or malignant cells, secreting cytokines, and regulating both innate and adaptive immune responses. ${ }^{30}$ The cross-communication between tumor and DCs results in the accumulation and expansion of regulatory $\mathrm{T}$ cells, which suppress the proliferation of other $\mathrm{T}$ cells in the tumor microenvironment through contact-dependent mechanisms, or independently through the secretion of IL-10 and transforming growth factor-beta. Regulatory $\mathrm{T}$ cells can also proceed to block the function of antitumor effector cells. ${ }^{31}$

In general, homeostasis disrupted by the progress of tumor development will activate immune cells, leading to the release of cytokines, growth factors, and enzymes, which will reestablish the extracellular matrix and contribute to further tumor progression. Meanwhile, the cross-communication between innate and adaptive immune systems is also distorted as a result of the continuous stimulation of certain inflammatory pathways. These processes play important roles in enhancing tumor cell survival, growth, and metastasis.

\section{Liposomes}

Liposomes in the nanometer scale appear as closed vesicles composed of lipid layers. ${ }^{32}$ Liposomes are biodegradable and can be used to encapsulate both hydrophilic and hydrophobic drugs. ${ }^{33}$ Various liposomal formulations have been developed to target malignant tissue. ${ }^{34}$ For example, liposomal doxorubicin (DOXIL ${ }^{\circledR}$, Janssen Biotech, Inc, Horsham, PA) is widely used in clinical settings for the treatment of Kaposi's sarcoma, ovarian cancer, and multiple myeloma. ${ }^{35,36}$ DOXIL $^{\circledR}$ is a stealth liposome formulation with a long blood circulation time. Although DOXIL ${ }^{\circledR}$ shows benefits in cancer patients, dose-limiting toxicities and doxorubicin-resistance have been observed. Intravenously administered unmodified liposomes are cleared quickly by the reticuloendothelial system (RES). ${ }^{37}$ Systemic delivery of cationic liposomes encounters obstacles such as the strong interaction with blood components, uptake by the RES, kidney filtration, and toxicity. ${ }^{38}$ If the agent to be delivered is targeted to organs other than the RES, modification of liposomes with polymers, such as 
Table I Strategies for targeting neovasculature endothelium and TAM utilizing liposomal nanoparticles

\begin{tabular}{|c|c|c|c|c|c|}
\hline Target & Tumor model & Therapeutic agent & Delivery system & Ligand/antibody & Reference \\
\hline $\begin{array}{l}\text { Breast cancer } \\
\text { stem cells }\end{array}$ & MCF-7 & Retinoic acid & DSPE PEG-2000: EPC LP & $\mathrm{N} / \mathrm{A}$ & 12 \\
\hline $\begin{array}{l}\text { Organ-specific } \\
\text { endothelial cells }\end{array}$ & N/A & Plasmid DNA & DOTIM-Chol-LP & N/A & 43 \\
\hline $\begin{array}{l}\text { Tumor } \\
\text { microvessels }\end{array}$ & A-Mel-3 & Paclitaxel & EndoTAG-I LP & $\mathrm{N} / \mathrm{A}$ & $45,46,48$ \\
\hline FGFR & $\mathrm{BI} 6-\mathrm{FIO}$ & Paclitaxel & DOTAP: Chol LP & tbFGF & 51 \\
\hline $\begin{array}{l}\text { Vascular } \\
\text { endothelium }\end{array}$ & N/A & $\begin{array}{l}\text { siRNA (Tie2, PKN3, } \\
\text { PTEN, CD3I) }\end{array}$ & Cationic LP AtuFECTOI & N/A & 54 \\
\hline L-VCAM-I & Colo 677 & $\mathrm{~N} / \mathrm{A}$ & soyPC: Chol: DSPE PEG-2000 LP & Anti-VCAM-I & 58 \\
\hline MTI-MMP & Colon $26 \mathrm{NL}-17$ & DPP-CNDAC & DSPC: Chol LP & GPLPLR & 62 \\
\hline MTI-MMP & HT I080 & Doxorubicin & HSPC: Chol LP & Anti-MTI-MMP & 63 \\
\hline Integrin $\alpha_{v} \beta 3$ & C26-colon carcinoma & Doxorubicin & DPPC: Chol: PEG-2000 LP & RGD-peptide & 68 \\
\hline Integrin $\alpha_{v}$ & A549 lung carcinoma & Paclitaxel & PC: Chol: DSPE PEG-2000 & RGD-peptide & 69 \\
\hline Integrin $\alpha 5 \beta$ I & MDA-MB-23I cells & Doxorubicin & PEG-2000 LP & PHSCNK & 71 \\
\hline Integrin $\alpha_{v} \beta 3$ & BI6 melanoma & $\mathrm{N} / \mathrm{A}$ & DSPC: DSPE PEG-2000 LP & C16Y peptide & 72 \\
\hline Integrin $\alpha_{v} \beta 3$ & $\mathrm{~B} 16-\mathrm{FIO}$ & $\mathrm{N} / \mathrm{A}$ & Gd-DTPA-DSA: DSPC: & RGD/Anx & 73 \\
\hline Galectin-I & melanoma & & Chol: DSPE PEG-2000 & & \\
\hline Aminopeptidase $\mathrm{N}$ & HT 1080 & c-myc siRNA & Liposome: polycation: DNA (LPD) & NGR peptide & 75 \\
\hline Aminopeptidase $\mathrm{N}$ & Orthotopic NB & Doxorubicin & HSPC: Chol: DSPE PEG-2000 & NGR peptide & 76 \\
\hline TAM & BI6-FI0 melanoma & $\begin{array}{l}\text { Clodronate, } \\
\text { prednisolone } \\
\text { phosphate }\end{array}$ & DPPC: Chol: DSPE PEG-2000 & $\mathrm{N} / \mathrm{A}$ & 83 \\
\hline p32/gClq receptor & SPC-AI & Doxorubicin & HSPC: Chol: DSPE-PEG & LyP-I & 87 \\
\hline CDI63 receptor & TAM & $\mathrm{N} / \mathrm{A}$ & HSPC: Chol: PE-PEG & $\begin{array}{l}\text { Anti-CDI63 } \\
\text { antibodies }\end{array}$ & 90 \\
\hline
\end{tabular}

Abbreviations: TAM, tumor-associated macrophage; N/A, not applicable; DSPE PEG-2000, distearoylphosphatidyl-ethanolamine-N-poly 2000; EPC LP, enzyme precipitating coated liposomes; DNA, deoxyribonucleic acid; DOTIM-Chol-LP, I-[2-[9-(Z)-octadecenoyloxy]]-2-[8](Z)heptadecenyl]-3[hydroxyethyl] imidazolinium chloride liposomes; FGFR, fibroblast growth factor receptors; DOTAP, N-[I-(2,3-Dioleoyloxy)propyl]-N,N,N-trimethylammonium methyl-sulfate; Chol LP, Chol liposome; tbFGF, truncated basic fibroblast growth factor; siRNA, small infecting ribonucleic acid; Tie2, PKN3, protein kinase-C molecule; PTEN, phosphatase and tensin homolog; CD3I, cluster of differentiation 3I; VCAM-I, vascular cell adhesion molecule-I; soyPC, hydrogenated soybean phosphatidycholine; MTI-MMP, membrane type-I matrix metalloproteases; DPP-CNDAC, 5'-O-dipalmitoylphosphatidyl 2'-C-cyano-2'-deoxy-I- 3 -D-arabino-pentofuranosylcytosine; DSPC, I,2-distearoyl-sn-glycero-3-phosphocholine; GPLPLR, stearoyl-Gly-Pro-Leu-Pro-Leu-Arg; HSPC, hydrogenated soy phosphatidylcholine; DPPC, Dipalmitoylphosphatidylcholine; RGD-peptide, Arginine-glycine-aspartic peptide; Gd-DTPA-DSA, Gadolinium diethylenetriaminepentaacetate-di(stearylamide); RDG/Anx, $\alpha_{v} \beta 3$ integrin-specific RGD ligand; LPD, liposome-polycation-DNA; NGR, Asn-GlyArg; NB, neobladder; SPC-AI, secretory pathway Ca2+-ATPase isoform I.

polyethylene glycol (PEG), can reduce the absorption of opsonins and avoid rapid RES clearance, thus achieving a long circulation. ${ }^{39}$

Binding of plasma proteins by liposomes is the primary mechanism for the RES to recognize circulating liposomes. ${ }^{38}$ Sterically stabilized liposomes extravasate through the gaps between the endothelial cells of the tumor vasculature (passive targeting) and collect in the interstitial space, where they are retained due to the lack of lymphatic clearance; this process is known as the enhanced permeation and retention (EPR) effect. ${ }^{40}$ Therefore, liposomes can deliver drugs at a much higher concentration in tumor tissues than in normal tissues. Liposomes can also be actively targeted to tumor tissues by recognizing specific tumor epitopes or receptors, which is achieved by coupling tumor-specific ligands or antibodies onto the surface of the liposomes, or by means of stimulus-sensitive drug carriers such as acid-triggered release, ${ }^{41}$ or enzymetriggered drug release. ${ }^{42}$ Both passive and active targeting can significantly reduce the toxic side effects of anticancer drugs and enhance the therapeutic efficacy of the drugs delivered.

\section{Delivery of liposomes to tumor microenvironment Targeting of endothelium in tumor neovasculature}

Tumors must induce the formation of new blood vessels in order to grow. Tumor blood vessels provide nutrients and oxygen, as well as eliminate waste from tumor tissues. ${ }^{7} \mathrm{New}$ blood vessel formation is based on a local shift in the balance between proangiogenic molecules (VEGF-A, FGF, and EGF) and antiangiogenic molecules (thrombospondin). This shift favors the initial phase of angiogenesis. Tumor blood vessels exhibit disorganized blood flow. Pericytes in the tumor endothelium are abnormal, which results in the leakiness in the tumor blood vessels as well, ${ }^{7}$ making the endothelium 
easily accessible by liposomal nanoparticles after intravenous administration. The tumor endothelium, which plays an important role in forming tumor neovasculature, has been the primary target in most of the liposomes reported to target anticancer drugs to the tumor microenvironment.

\section{Targeting of endothelial cells using positively charged liposomal nanoparticles}

Positively charged liposomes have been investigated to deliver drugs to tumor blood vessels. The negatively charged proteoglycans on the endothelial cell membrane could bind and help cells internalize cationic liposomes due to their electrostatic interaction. Mclean et $\mathrm{al}^{43}$ showed that cationic 1-[2-[9-(Z)-octadecenoyloxy]]-2-[8](Z)heptadecenyl]-3[hydroxyethyl] imidazolinium chloride (DOTIM) liposomes loaded with DNA accumulated in the vasculature after intravenous injection into mice, demonstrating that positively charged liposomes can be taken up by the normal endothelium. This finding triggered a series of studies that focused on the targeting of the tumor neovasculature.

To enhance the targeting efficiency to tumor blood vessels, positively charged protamine was injected intravenously before the injection of cationic liposomes, resulting in a 2.2-fold increase in the accumulation of liposomes in tumor vasculature. ${ }^{44}$ Importantly, it was found that the quiescent endothelium took up less of the liposomes than the tumor endothelium. It was thought that protamine functioned by saturating areas of high uptake outside the tumors or by changing the tumor microenvironment to facilitate the interaction between endothelium and cationic liposomes. ${ }^{44}$

The therapeutic efficacy of cationic liposomes encapsulated with paclitaxel has been extensively examined..$^{45-47}$ The paclitaxel-loaded cationic liposomes inhibit tumor growth and metastasis, and thereby prolong the survival of animals. They target tumor neovasculature and increase tumor microvessel leakiness, leading to vascular damage. The observed antiangiogenic mechanisms of the cationic paclitaxel liposomes include reduced blood vessel density in the tumor periphery and reduced endothelial cell proliferation. In addition, Strieth et $\mathrm{al}^{45,46}$ found that platelet activation within the tumor microcirculation contributed to the effects of the cationic liposomes encapsulated with paclitaxel, and the accumulation of platelets reduced tumor capillary blood flow, giving rise to reduced tumor perfusion.

EndoTAG- ${ }^{\circledR}$ (ET; Medigene AG, Martinsried, Germany) cationic liposomes loaded with paclitaxel were found to exhibit antitumor activity by targeting tumor endothelial cells in solid tumors. ${ }^{48}$ Patients with advanced cancer and liver metastasis were treated with ET after single or repeated doses (22 $\mathrm{mg} / \mathrm{m}^{2}$, intravenously) the tumor vasculature or pharmacokinetic effects were evaluated. The pharmacokinetic profile, which was measured by dynamic contrast-enhanced magnetic resonance imaging and contract-enhanced ultrasound, showed slight accumulation of paclitaxel in tumor tissues after repeated doses. The pharmacokinetic parameters were similar to the liposomal paclitaxel. ${ }^{48}$ ET liposomes were well tolerated, as $50 \%$ of the patients had stable disease after the first treatment cycle. ${ }^{48}$

Fibroblast growth factor receptors (FGFR) are reported to be overexpressed on the surface of tumor neovasculature, as well as in a variety of tumor cells. ${ }^{49,50}$ Wang et $\mathrm{al}^{51}$ used truncated basic fibroblast growth factor (bFGF)-conjugated cationic liposomes to improve the biodistribution and pharmacokinetics of paclitaxel in tumor-bearing mice. In their study, the pharmacokinetics and tissue distribution of paclitaxel in truncated bFGF-conjugated cationic liposomes (tbFGF-LPs-PTX) and tbFGF-free cationic liposomal paclitaxel (LPs-PTX) were evaluated in tumor-bearing mice and compared to that of free paclitaxel (F-PTX). The tbFGF-LPs-PTX and the LPs-PTX exhibited similar pharmacokinetic characteristics, but the biodistribution of the tbFGF-LPs-PTX was different from that of the LP-PTX, with the tbFGF-LPs-PTX accumulated more extensively in tumors and spleen. ${ }^{51}$ The tbFGF-LPs-PTX effectively targeted the paclitaxel to tumors and prolonged the survival time of tumor-bearing mice..$^{51}$

Finally, lipoplexes formed by the self-assembly of nucleic acids and positively charged liposomes were applied in many studies to deliver oligonucleotides or plasmids to tumor blood vessel endothelium. ${ }^{52,53}$ Santel et al $^{54}$ showed that liposomes prepared with a mixture of cationic and fusogenic lipids, and complexed with negatively charged small interfering ribonucleic acid effectively targeted the small interfering ribonucleic acid to tumor vascular endothelium. After intravenous injection, endothelial cell-specific uptake was confirmed in the tumor tissue, and the silencing of endothelium-specific genes encoding of CD31 and Tie 2 was observed. ${ }^{54}$

\section{Ligand- or antibody-conjugated liposomal nanoparticles}

Besides the positively charge-based targeting, most of the liposomal targeting strategies focus on targeting specific receptors overexpressed on tumor endothelial cells. ${ }^{55}$ Decades of investigations on the molecular basis of angiogenesis have led to the discovery of several proteins expressed on solid tumor-associated angiogenic vessels, 
while they are barely detectable in established blood vessels. Examples of such proteins include $\alpha_{\mathrm{v}}$ integrins, the receptor for angiogenic growth factor, and other types of membranespanning molecules, such as transmembrane glycoprotein and aminopeptidase $\mathrm{N}$.

Vascular cell adhesion molecule-1 (VCAM-1), an immunoglobulin-like transmembrane glycoprotein, is a surface receptor that is overexpressed on tumor blood vessels. ${ }^{56}$ It is also found in other cell types such as macrophages, DCs, and myoblasts, but to a lesser extent. VCAM-1 can generate firm cell-cell adhesion. VCAM-1-targeted liposomes are promising in the treatment of cancer by altering endothelial function. Voinea et $\mathrm{al}^{57}$ showed that anti-VCAM-1 coupled liposomes bind selectively and specifically to tumor necrosis factor-alpha (TNF- $\alpha$ )-activated human endothelial cells in vitro. Gosk et $\mathrm{al}^{58}$ also demonstrated tumor-specific targeting of liposomes surface-conjugated with anti-VCAM-1. In vitro the VCAM-1-targeted liposomes showed specific binding to activated endothelial cells with or without simulated blood flow. In vivo tumor targeting was studied in mice with Colo 677 xenografts, and more anti-VCAM-1 liposomes accumulated in tumor blood vessels than control liposomes. ${ }^{58}$

Metastatic invasion is advanced by the attachment of tumor cells to the extracellular matrix and the degradation of matrix components by tumor-associated proteases. ${ }^{33}$ Matrix metalloproteases (MMPs) are a family of enzymes that degrade the basement membrane and extracellular matrix, leading to tissue remodeling. Membrane type-1-MMP (MT1MMP) is expressed specifically on vascular endothelial cells as well as in tumor cells. ${ }^{59}$ It is a membrane-anchored enzyme and is involved in the degradation of various extracellular membrane components such as collagen, fibronectin, laminin, fibrin, aggrecan, and vitronectin. In addition, MT1-MMP can activate several promatrix metalloproteinases, which enhance its role in connective tissue remodeling, especially for angiogenesis. ${ }^{59-61}$ Therefore, any agent that has a high affinity to MT1-MMP may potentially be used for the active targeting of tumor angiogenic vessels. Kondo et $\mathrm{al}^{62}$ investigated the targeting of angiogenic endothelium and tumor cells using stearoyl-Gly-Pro-Leu-Pro-Leu-Arg liposomes (GPLPLRLip). PLPL was found to be a consensus substrate sequence for MT1-MMP. ${ }^{61}$ In vitro, GPLPLR-Lip showed a stronger binding to human umbilical vein endothelial cells, as compared to unmodified liposomes. ${ }^{62}$ In vivo, such modification enhanced tumor accumulation of the liposomes by fourfold, and tumor growth was strongly suppressed by GPLPLRliposomes loaded with the nucleoside analogue $5^{\prime}$-O-dipalmitoylphosphatidyl 2'-C-cyano-2'-deoxy-1-beta-d-arabino-pen tofuranosylcytosine. ${ }^{62}$ Finally, it was also demonstrated that anti-MT1-MMP antibody-modified liposomes significantly inhibited tumor progression, as compared to unmodified ones. Hatakeyama et al ${ }^{63}$ coupled the Fab' fragment of antiMT1-MMP antibodies to PEG-liposomes encapsulated with doxorubicin. In HT 1080 cells, which express a high level of MT1-MMP, the anti-MT1-MMP Fab' fragment significantly enhanced the cellular uptake of the liposomes. ${ }^{63}$

The transmembrane glycoprotein integrins had served as one of the most important targets for active targeting. Integrins mediate cell-to-cell and cell-to-matrix interactions, and play an important role in various vital cellular functions, including signal transduction, gene expression, cell proliferation, apoptosis, invasion, metastasis, angiogenesis, and tumor growth. ${ }^{64,65}$ Integrin $\alpha_{\mathrm{v}} \beta 3, \alpha_{\mathrm{v}} \beta 5$, and $\alpha 5 \beta 1$ have been identified on tumor neovascular endothelial cells and some tumor cells. ${ }^{66}$ It was demonstrated in many studies that activated endothelial cells bind and internalize PEG-liposomes modified with cyclic RGD-peptides on their surface. RGDpeptides have a high affinity to integrin $\alpha_{v} \beta 3 .{ }^{67}$ In vitro RGD-peptide-modified liposomes loaded with doxorubicin were found to be significantly more cytotoxic to endothelial cells than unmodified control liposomes. In vivo, in a doxorubicin-resistant murine C26-colon carcinoma model, only the RGD-liposomes loaded with doxorubicin inhibited tumor progression, whereas control doxorubicin-loaded RADliposomes and nontargeted PEG-liposomes failed. Since C26-tumor cells are highly resistant to doxorubicin, it was thought that the RGD-liposomes achieved antitumor effects through their cytotoxic action on angiogenic endothelium. ${ }^{68}$ Paclitaxel has also been tested in such a setting as well. ${ }^{69}$ In mice with A549 xenografts, tumors treated with the cyclic RGD-liposomes loaded with paclitaxel showed a lower tumor micro-vessel density than tumors treated with nontargeted paclitaxel liposomes, which was explained by the specific recognition and binding of the cyclic RGD-liposomes to the endothelial cells, resulting in an increased local paclitaxel concentration in tumor tissues. ${ }^{69}$

Integrin $\alpha 5 \beta 1$ plays a more significant role than $\alpha_{v} \beta 3$ in tumor angiogenesis. It is not expressed in quiescent vascular endothelial cells, but is highly expressed in angiogenic endothelial cells and certain tumor cells. ${ }^{70}$ ATN-161 ( $\mathrm{N}$-acetyl-proline-histidine-serine-cysteine-asparagineamide, PHSCN) is a ligand of integrin $\alpha 5 \beta 1$. Dai et $\mathrm{al}^{71}$ used PHSCN-conjugated PEG-liposomes loaded with doxorubicin to target both tumor angiogenesis and tumor cells, and demonstrated that the PHSCNK significantly enhanced the cell uptake and cytotoxicity of the doxorubicin-loaded 
liposomes by both human umbilical vein endothelial cells (HUVEC) and breast cancer cells through integrin-mediated endocytosis. $^{71}$

Hamano et $\mathrm{al}^{72}$ investigated PEGylated liposomes modified with $\mathrm{C} 16 \mathrm{Y}$ peptide, which is derived from the laminin $\gamma 1$ chain and binds to integrins $\alpha_{v} \beta 3$ and $\alpha 5 \beta 1$, targeting both HUVECs and B16 cells, which are representative of endothelial and cancer cells, respectively. The results indicate that the $\mathrm{C} 16 \mathrm{Y}$ peptide-mediated PEG-liposome can enhance the uptake of both HUVECs and B16 cells, and that their uptake was dependent on temperature, which points to the involvement of receptor-mediated endocytosis. Kluza et $\mathrm{al}^{73}$ improved activated targeting to endothelium in tumor neovasculature by incorporation of two angiogenesis ligands. A dual-conjugated liposome containing galectin-1specific anginex (Anx) and the $\alpha_{v} \beta 3$ integrin-specific RGD ligand (Anx/RGD-Lps) were evaluated in mice bearing B16F10 melanoma to investigate the targeting properties and clearance kinetics of Anx/RGD-Lps. ${ }^{73}$ Fluorescence microscopy was used to access the specificity of Anx/ RGD-Lps associated with tumor endothelium, and found it to be $53 \% \pm 6 \%$, which was significantly higher compared to single targeting liposomes Anx-L 43\% $\pm 9 \%$ or RGF-L $28 \% \pm 8 \%$. The synergistic targeting of galectin- 1 and $\alpha_{v} \beta 3$ improved the specificity of the liposomal nanoparticles to tumor endothelium. ${ }^{73}$

Aminopeptidase $\mathrm{N}$ is a protease overexpressed on membranes of endothelial cells of angiogenic vessels in solid tumors, and the aspargine-glycine-arginine (NGR)-motif is one of the various ligands identified thus far that promote peptide binding to aminopeptidase N. ${ }^{74,75}$ Pastorino et $\mathrm{al}^{76}$ coupled peptides containing NGR-motif to the surface of PEG-liposomes loaded with doxorubicin. In severe combined immunodeficiency mice with an orthotopic neuroblastoma, NGR-peptide-conjugated liposomes achieved a tenfold increase in tumor accumulation, as compared to conventional NGR-peptide-free liposomes. ${ }^{76}$ Such accumulation can be blocked by coinjection of free NGR, indicating the NGRspecific uptake of the liposomes. ${ }^{76}$

\section{Targeting tumor-associated macrophages}

"Classically activated" macrophages release proinflammatory cytokines such as TNF- $\alpha$, IL- $1 \beta$, IL-12, and enhanced the expression of major histocompatibility complex-2, CD80, and CD86, which are important for antigen presentation. This process has been utilized in drug targeting. Macrophages can be targeted and activated to confer tumor suppressive properties in cancer treatment. ${ }^{77,78}$ Activation of macrophages results in augmenting antitumor immune responses by the induction of proinflammatory mediators such as TNF- $\alpha$, IL-8, and nitric oxide. ${ }^{79,80}$

During tumor initiation and progression, tumor tissues promote an inflammatory environment that supports tumor growth. Macrophages are one of those mononuclear phagocytes significantly accumulated in tumors. TAMs have a distinct phenotype, compared with "classically activated" macrophages. TAMs are significantly active in angiogenesis, tissue remodeling, and repair. ${ }^{81}$ TAMs also produce less proinflammatory cytokines and overexpress surface scavenger receptor A and mannose receptor. ${ }^{82}$ Moreover, TAMs have decreased capacity to act as antigen-presenting cells.

PEG-liposomes loaded with prednisolone phosphate (LPsPLP), which is selectively toxic to macrophages, were investigated in B16-F10 melanoma-bearing mice. LPs-PLP exerted antitumor activity through the inhibition of tumor angiogenesis by the depletion of TAMs. LPs-PLP reduced TAM-mediated production of proangiogenic factors such as granulocytemacrophage colony-stimulating factor, macrophage colonystimulating factor, IL-1 $\alpha$, IL-1 $\beta$, IL-6, and IL-9, but the production of antiangiogenic factors is hardly affected..$^{83}$

The therapeutic efficacy of DOXIL ${ }^{\circledR}$ was evaluated in B16-F10 melanoma-bearing mice in the presence or absence of TAMs ${ }^{84}$ which was controlled using PEG-liposomes loaded with clodronate injected 24 hours before the actual treatment. PEG-liposomes loaded with clodronate suppressed tumor progression through the reduction of the proangiogenic function of TAMs. The antitumor effect of DOXIL ${ }^{\circledR}$ was only partially due to its ability to inhibit TAM-mediated angiogenesis.

The LyP-1 peptide has been shown to have high association to lymphatic vessels and tumor cells. ${ }^{85}$ In particular, it was shown to selectively bind to $\mathrm{p} 32 / \mathrm{gC} 1 \mathrm{q}-$ a receptor overexpressed in tumor cells. ${ }^{86} \mathrm{LyP}-1$ was found to accumulate in hypoxic areas in the tumor, and thus its potential as a target for tumor therapy was evaluated. The LyP-1was conjugated to the PEGylated liposome surface to investigate the targeted therapy ${ }^{87}$ In vitro LyP-1-conjugated PEG-liposomes had higher uptake by SPC-A1 lung adenocarcinoma cells, but not by normal lymph nodes. In vivo near-infrared fluorescence imaging results indicated that LyP-1-mediated PEG-liposomes enhanced the liposome uptake by metastatic lymph nodes, but not by normal lymph nodes. Moreover, immunofluorescence showed that LyP-1-mediated PEG-liposomes were specifically distributed adjacent to both tumor lymphatics and TAMs in metastatic lymph nodes. Further, a pharmacodynamic study comparing unmodified liposomes to LyP-1-mediated 
doxorubicin-loaded PEG-liposomes showed enhanced inhibition of tumor cells in vitro, and lymphatic metastatic tumor in vivo with the targeting motif. ${ }^{87}$

The hemoglobin scavenger receptor CD163 is overexpressed in the tissues of resident macrophages of the M2 phenotype, and in macrophages in sites of inflammation and tumor growth. ${ }^{88,89}$ Etzerodt et $\mathrm{al}^{90}$ investigated the CD163binding monoclonal antibodies coupled to the surface of PEGliposomes to target the CD163 cells and macrophages. The antibody-mediated PEG-liposomes significantly increased the uptake of liposomes in both CD163 transfected cells and macrophages. Further the receptor CD163-targeted PEGliposomal doxorubicin exhibited strong cytotoxic effects in CD163-expressing human monocytes. ${ }^{90}$ The CD163-binding monoclonal antibodies-mediated PEG-liposome is a potential approach to target therapeutic agents to macrophages that support inflammatory and malignant progression.

Finally, Matsui et $\mathrm{al}^{77}$ had used oligomannose-coated liposomes to successfully deliver 5-fluorouracil to peritoneal macrophages, which then delivered the 5-fluorouracil to omental milky spots, known as the initial metastatic sites in the peritoneal cavity. ${ }^{91}$ At Table 1 we summarized strategies for targeting neovasculature endothelium and TAM utilizing liposomal nanoparticles.

\section{Targeting tumor-associated fibroblasts}

In addition to endothelial cells and macrophages, fibroblasts are another major component of tumor microenvironment. Fibroblast activation protein (FAP) is a surface antigen selectively expressed by reactive tumor-associated fibroblast (TAF). Liposomes coupled with FAP antibody Fv fragments were internalized into the endosomal compartment of FAPexpressing cells, as shown by Baum et al. ${ }^{92}$ The value of such an approach needs to be confirmed in vivo.

Sadlonova et $\mathrm{al}^{93}$ compared gene expression profiles of fibroblasts extracted from breast tumors with those from normal breast tissue. A total of 420 genes were found to be differentially expressed, of which 240 genes were overexpressed by TAF. These genes are relevant to the fibroblast-epithelial interaction. They are also potential ligands for TAF-specific delivery of liposomes for cancer treatment.

\section{Targeting tumor-associated pericytes}

Pericytes have been found to be involved in triggering metastasis by perturbing pericyte-endothelial cell-cell interactions. Pericyte dysfunction resulted in metastasis in distant organs and local lymph nodes. ${ }^{94}$ Platelet-derived growth factor receptor-beta (PDGFR- $\beta$ ) is overexpressed on tumor-associated pericytes and fibroblasts, while it is expressed on malignant cells. ${ }^{95}$ Human serum albumin was modified with a cyclic peptide as ligand-binding to PDGFR- $\beta$ to form a carrier, which was loaded with doxorubicin to target tumors. ${ }^{96}$ The data showed that the targeted doxorubicin-carrier accumulated in PDGFR- $\beta$ expressing vascular pericytes and stromal fibroblasts, in addition to malignant cells, while it appeared to be rapidly clearing from other organs. Treatment with the targeted carrier loaded with doxorubicin markedly reduced the tumor growth without showing doxorubicin-related side effects. ${ }^{96}$ The investigation indicated that this is a novel strategy to target more cell types simultaneously for good anticancer potential. The ligand-mediated liposomal anticancer agent may be a promising approach to achieve this goal.

\section{Targeting tumor-associated extracellular matrix}

The extracellular matrix surrounding malignant cells is significantly different from that surrounding normal cells in tumor tissue. In particular, tenascin-C (TN-C) as a multifunctional extracellular matrix glycoprotein is overexpressed in many different tumors such as gliomas, breast tumors, and prostate tumors. ${ }^{97}$ The sulfatide is a natural acidic glycosphingolipid that can be utilized as a ligand of targeted liposomes via binding to TN-C. The sulfatide-mediated liposomes were internalized by both caveolae/lipid raft- and clathrin-dependent pathways in experiments with TN-C-expressing glioma cells..$^{98}$ These studies exhibited a new strategy for more effective anticancer chemotherapeutics.

\section{Targeting to tumor-associated lymphocytes}

The lymphatic system is involved in the metastasis of most human cancers such as breast, ovarian, lung, colon, and prostate cancer. ${ }^{99}$ Exploration exhibited that VEGF-C and VEGF-D enhanced lymphangiogenesis, the metastatic spread of malignant cells to lymph nodes. ${ }^{100,101}$ Honokiol is a potential antiangiogenic and antitumor medicine that acts by blocking VEGF-induced autophosphorylation of VEGF receptor 2. To investigate related lymph node metastasis and antitumor activity, liposomal honokiol was injected intraperitoneally in a mouse model of xenografted Lewis lung carcinoma cells overexpressing VEGF-D. Liposomal honokiol markedly reduced metastasis to the lymph nodes when compared with the control group. The decrease of lymphatic metastasis to the lymph nodes depended on the administered doses (30\% and $10 \%$ for $12.5 \mathrm{mg} / \mathrm{Kg} /$ day and $50 \mathrm{mg} / \mathrm{Kg} /$ day, respectively). ${ }^{101}$ 


\section{Conclusion and future perspectives}

The dynamic interactions between tumor cells and cells in the tumor microenvironment coordinate events that are critical for tumor progression and metastasis formation. The complex tumor microenvironment calls for a drug delivery system that can target tumor cells as well as nontumor cells. Liposomal nanoparticles demonstrated immense potential in effectively and safely targeting anticancer drugs to tumors. PEGylation of liposomes can help decrease the rapid clearance of the liposomes from the circulation and increase the accumulation of the liposomes in tumor tissues by the EPR effect. Cationic liposomes are effective in delivering anticancer drugs to tumor tissues by targeting the negatively charged proteoglycans on the membrane of the endothelial cells in tumor neovasculature. Liposomes that are surface-conjugated with ligands, or antibodies against surface molecules that are specific or overexpressed on tumor-associated cells, can actively and selectively target anticancer drugs to tumors.

The translation of the success of the aforementioned liposomes that target tumor microenvironments ranging from animal studies to clinics will be dependent on their ability to target all aspects of the tumor microenvironment, including cancer stem cells. The complex nature of tumor growth and progression dictates that a curative therapy needs to expand from aiming at single pathways to addressing multiple pathways simultaneously. Therefore, future tumor-targeting (liposomal) nanoparticles need to be carefully engineered to not only kill tumor cells, but also to modify the tumor microenvironment so that it is unfavorable for tumor cells to grow, and for cancer stem cells to repopulate.

\section{Acknowledgments}

This manuscript was written by Professor Gang Zhao when he was a visiting professor in the Pharmaceutics Division at The University of Texas at Austin College of Pharmacy. He was supported by a Shandong Provincial Natural Science Foundation grant (Y2008C03) and a scholarship from Shandong Province, China. We would like to thank Dr Zhengrong Cui for useful discussions and initial editing of the review.

\section{Disclosure}

The authors report no conflicts of interest in this work.

\section{References}

1. Jemal A, Bray F, Center MM, Ferlay J, Ward E, Forman D. Global cancer statistics. CA Cancer J Clin. 2011;61(2):69-90.

2. De Palma M, Lewis CE. Cancer: Macrophages limit chemotherapy. Nature. 2011;472(7343):303-304.

3. Arias JL. Drug targeting strategies in cancer treatment: an overview. Mini Rev Med Chem. 2011;11(1):1-17.
4. Gottesman MM. Mechanisms of cancer drug resistance. Annu Rev Med. 2002;53:615-627.

5. Bindra RS, Glazer PM. Genetic instability and the tumor microenvironment: towards the concept of microenvironment-induced mutagenesis. Mutat Res. 2005;569(1-2):75-85.

6. Reynolds TY, Rockwell S, Glazer PM. Genetic instability induced by the tumor microenvironment. Cancer Res. 1996;56(24):5754-5757.

7. Hida K, Kawamoto T, Ohga N, Akiyama K, Hida Y, Shindoh M. Altered angiogenesis in the tumor microenvironment. Pathol Int. 2011;61(11):630-637.

8. Wojtkowiak JW, Verduzco D, Schramm KJ, Gillies RJ. Drug resistance and cellular adaptation to tumor acidic $\mathrm{pH}$ microenvironment. $\mathrm{Mol}$ Pharm. 2011;8(6):2032-2038.

9. Liotta LA, Kohn EC. The microenvironment of the tumour-host interface. Nature. 2001;411(6835):375-379.

10. Weber CE, Kuo PC. The tumor microenvironment. Surg Oncol. 2011;21(3):172-177.

11. Ungefroren H, Sebens S, Seidl D, Lehnert H, Hass R. Interaction of tumor cells with the microenvironment. Cell Commun Signal. 2011;9:18.

12. Li RJ, Ying X, Zhang Y, et al. All-trans retinoic acid stealth liposomes prevent the relapse of breast cancer arising from the cancer stem cells. J Control Release. 2011;149(3):281-291.

13. Kerbel RS. Tumor angiogenesis. N Engl J Med. 2008;358(19): 2039-2049.

14. Nagy JA, Chang SH, Dvorak AM, Dvorak HF. Why are tumour blood vessels abnormal and why is it important to know? $\mathrm{Br} J$ Cancer. 2009;100(6):865-869.

15. Carmeliet P. Angiogenesis in life, disease and medicine. Nature. 2005;438(7070):932-936

16. Gerhardt H, Semb H. Pericytes: gatekeepers in tumour cell metastasis? J Mol Med (Berl). 2008;86(2):135-144.

17. Dvorak HF, Weaver VM, Tlsty TD, Bergers G. Tumor microenvironment and progression. J Surg Oncol. 2011;103(6):468-474.

18. Avraamides CJ, Garmy-Susini B, Varner JA. Integrins in angiogenesis and lymphangiogenesis. Nat Rev Cancer. 2008;8(8):604-617.

19. Nagy JA, Dvorak AM, Dvorak HF. VEGF-A and the induction of pathological angiogenesis. Annu Rev Pathol. 2007;2:251-275.

20. Ribatti D, Vacca A. The role of microenvironment in tumor angiogenesis. Genes Nutr. 2008;3(1):29-34.

21. de Visser KE, Eichten A, Coussens LM. Paradoxical roles of the immune system during cancer development. Nat Rev Cancer. 2006;6(1):24-37.

22. Lewis CE, Pollard JW. Distinct role of macrophages in different tumor microenvironments. Cancer Res. 2006;66(2):605-612.

23. Leek RD, Lewis CE, Whitehouse R, Greenall M, Clarke J, Harris AL. Association of macrophage infiltration with angiogenesis and prognosis in invasive breast carcinoma. Cancer Res. 1996;56(20): 4625-4629.

24. DeNardo DG, Andreu P, Coussens LM. Interactions between lymphocytes and myeloid cells regulate pro- versus anti-tumor immunity. Cancer Metastasis Rev. 2010;29(2):309-316.

25. Andreu P, Johansson M, Affara NI, et al. FcRgamma activation regulates inflammation-associated squamous carcinogenesis. Cancer Cell. 2010;17(2):121-134.

26. Allavena P, Sica A, Garlanda C, Mantovani A. The Yin-Yang of tumor-associated macrophages in neoplastic progression and immune surveillance. Immunol Rev. 2008;222:155-161.

27. Bingle L, Brown NJ, Lewis CE. The role of tumour-associated macrophages in tumour progression: implications for new anticancer therapies. J Pathol. 2002;196(3):254-265.

28. Qian BZ, Pollard JW. Macrophage diversity enhances tumor progression and metastasis. Cell. 2010;141(1):39-51.

29. Wai LE, Garcia JA, Martinez OM, Krams SM. Distinct roles for the NK cell-activating receptors in mediating interactions with dendritic cells and tumor cells. J Immunol. 2011;186(1):222-229.

30. Caligiuri MA. Human natural killer cells. Blood. 2008;112(3):461-469. 
31. Ferrone $S$, Whiteside TL. Tumor microenvironment and immune escape. Surg Oncol Clin N Am. 2007;16(4):755-774, viii.

32. Haley B, Frenkel E. Nanoparticles for drug delivery in cancer treatment. Urol Oncol. 2008;26(1):57-64.

33. Penate Medina O, Haikola M, Tahtinen M, et al. Liposomal tumor targeting in drug delivery utilizing MMP-2- and MMP-9-binding ligands. J Drug Deliv. 2011;2011:160515.

34. Musacchio T, Torchilin VP. Recent developments in lipid-based pharmaceutical nanocarriers. Front Biosci. 2011;16:1388-1412.

35. Pérez-López ME, Curiel T, Gómez JG, Jorge M. Role of pegylated liposomal doxorubicin (Caelyx) in the treatment of relapsing ovarian cancer. Anticancer Drugs. 2007;18(5):611-617.

36. Ludwig H, Strasser-Weippl K, Schreder M, Zojer N. Advances in the treatment of hematological malignancies: current treatment approaches in multiple myeloma. Ann Oncol. 2007;18 Suppl 9:ix64-ix70.

37. Moghimi SM, Szebeni J. Stealth liposomes and long circulating nanoparticles: critical issues in pharmacokinetics, opsonization and protein-binding properties. Prog Lipid Res. 2003;42(6):463-478.

38. Guo X, Huang L. Recent advances in nonviral vectors for gene delivery. Acc Chem Res. 2012;45(7):971-979.

39. Romberg B, Hennink WE, Storm G. Sheddable coatings for longcirculating nanoparticles. Pharm Res. 2008;25(1):55-71.

40. Lasic DD. Doxorubicin in sterically stabilized liposomes. Nature. 1996;380(6574):561-562.

41. Kim IY, Kang YS, Lee DS, et al. Antitumor activity of EGFR targeted $\mathrm{pH}$-sensitive immunoliposomes encapsulating gemcitabine in A549 xenograft nude mice. J Control Release. 2009;140(1):55-60.

42. Andresen TL, Jensen SS, Kaasgaard T, Jørgensen K. Triggered activation and release of liposomal prodrugs and drugs in cancer tissue by secretory phospholipase A2. Curr Drug Deliv. 2005;2(4): 353-362.

43. McLean JW, Fox EA, Baluk P, et al. Organ-specific endothelial cell uptake of cationic liposome-DNA complexes in mice. Am J Physiol. 1997;273(1 Pt 2):H387-H404.

44. Eichhorn ME, Strieth S, Krasnici S, et al. Protamine enhances uptake of cationic liposomes in angiogenic microvessels. Angiogenesis. 2004;7(2):133-141.

45. Strieth S, Eichhorn ME, Werner A, et al. Paclitaxel encapsulated in cationic liposomes increases tumor microvessel leakiness and improves therapeutic efficacy in combination with Cisplatin. Clin Cancer Res. 2008;14(14):4603-4611.

46. Strieth S, Nussbaum CF, Eichhorn ME, et al. Tumor-selective vessel occlusions by platelets after vascular targeting chemotherapy using paclitaxel encapsulated in cationic liposomes. Int $J$ Cancer. 2008;122(2):452-460.

47. Schmitt-Sody M, Strieth S, Krasnici S, et al. Neovascular targeting therapy: paclitaxel encapsulated in cationic liposomes improves antitumoral efficacy. Clin Cancer Res. 2003;9(6):2335-2341.

48. Fasol U, Frost A, Büchert M, et al. Vascular and pharmacokinetic effects of EndoTAG-1 in patients with advanced cancer and liver metastasis. Ann Oncol. 2012;23(4):1030-1036.

49. Danielsen T, Rofstad EK. VEGF, bFGF and EGF in the angiogenesis of human melanoma xenografts. Int J Cancer. 1998;76(6):836-841.

50. Kwabi-Addo B, Ozen M, Ittmann M. The role of fibroblast growth factors and their receptors in prostate cancer. Endocr Relat Cancer. 2004;11(4):709-724.

51. Wang X, Deng L, Chen X, et al. Truncated bFGF-mediated cationic liposomal paclitaxel for tumor-targeted drug delivery: improved pharmacokinetics and biodistribution in tumor-bearing mice. J Pharm Sci. 2011;100(3):1196-1205.

52. Spelios M, Kearns M, Savva M. From gene delivery to gene silencing: plasmid DNA-transfecting cationic lipid 1,3-dimyristoylamidopropane2-[bis(2-dimethylaminoethane)] carbamate efficiently promotes small interfering RNA-induced RNA interference. Biochemistry. 2010;49(27):5753-5759.

53. Jeong JH, Park TG, Kim SH. Self-assembled and nanostructured siRNA delivery systems. Pharm Res. 2011;28(9):2072-2085.
54. Santel A, Aleku M, Keil O, et al. A novel siRNA-lipoplex technology for RNA interference in the mouse vascular endothelium. Gene Ther. 2006;13(16):1222-1234.

55. Murase Y, Asai T, Katanasaka Y, et al. A novel DDS strategy, "dualtargeting", and its application for antineovascular therapy. Cancer Lett. 2010;287(2):165-171.

56. Osborn L, Hession C, Tizard R, et al. Direct expression cloning of vascular cell adhesion molecule 1, a cytokine-induced endothelial protein that binds to lymphocytes. Cell. 1989;59(6):1203-1211.

57. Voinea M, Manduteanu I, Dragomir E, Capraru M, Simionescu M. Immunoliposomes directed toward VCAM-1 interact specifically with activated endothelial cells - a potential tool for specific drug delivery. Pharm Res. 2005;22(11):1906-1917.

58. Gosk S, Moos T, Gottstein C, Bendas G. VCAM-1 directed immunoliposomes selectively target tumor vasculature in vivo. Biochim Biophys Acta. 2008;1778(4):854-863.

59. Handsley MM, Edwards DR. Metalloproteinases and their inhibitors in tumor angiogenesis. Int J Cancer. 2005;115(6):849-860.

60. Itoh Y, Seiki M. MT1-MMP: a potent modifier of pericellular microenvironment. J Cell Physiol. 2006;206(1):1-8.

61. Barbolina MV, Stack MS. Membrane type 1-matrix metalloproteinase: substrate diversity in pericellular proteolysis. Semin Cell Dev Biol. 2008;19(1):24-33.

62. Kondo M, Asai T, Katanasaka Y, et al. Anti-neovascular therapy by liposomal drug targeted to membrane type-1 matrix metalloproteinase. Int J Cancer. 2004;108(2):301-306.

63. Hatakeyama $\mathrm{H}$, Akita $\mathrm{H}$, Ishida $\mathrm{E}$, et al. Tumor targeting of doxorubicin by anti-MT1-MMP antibody-modified PEG liposomes. Int $J$ Pharm. 2007;342(1-2):194-200.

64. Hynes RO. Integrins: versatility, modulation, and signaling in cell adhesion. Cell. 1992;69(1):11-25.

65. Hood JD, Cheresh DA. Role of integrins in cell invasion and migration. Nat Rev Cancer. 2002;2(2):91-100.

66. Serini G, Valdembri D, Bussolino F. Integrins and angiogenesis: a sticky business. Exp Cell Res. 2006;312(5):651-658.

67. Janssen AP, Schiffelers RM, ten Hagen TL, et al. Peptide-targeted PEG-liposomes in anti-angiogenic therapy. Int J Pharm. 2003;254(1): $55-58$.

68. Schiffelers RM, Koning GA, ten Hagen TL, et al. Anti-tumor efficacy of tumor vasculature-targeted liposomal doxorubicin. J Control Release. 2003;91(1-2):115-122.

69. Meng S, Su B, Li W, et al. Integrin-targeted paclitaxel nanoliposomes for tumor therapy. Med Oncol. 2011;28(4):1180-1187.

70. Kim S, Bell K, Mousa SA, Varner JA. Regulation of angiogenesis in vivo by ligation of integrin alpha5beta1 with the central cell-binding domain of fibronectin. Am J Pathol. 2000;156(4):1345-1362.

71. Dai W, Yang T, Wang X, Wang J, Zhang X, Zhang Q. PHSCNKModified and doxorubicin-loaded liposomes as a dual targeting system to integrin-overexpressing tumor neovasculature and tumor cells. J Drug Target. 2010;18(4):254-263.

72. Hamano N, Negishi Y, Fujisawa A, et al. Modification of the C16Y peptide on nanoparticles is an effective approach to target endothelial and cancer cells via the integrin receptor. Int J Pharm. 2012;428(1-2): $114-117$.

73. Kluza E, Jacobs I, Hectors SJ, et al. Dual-targeting of $\alpha v \beta 3$ and galectin-1 improves the specificity of paramagnetic/fluorescent liposomes to tumor endothelium in vivo. J Control Release. 2012;158(2): 207-214.

74. Curnis F, Sacchi A, Corti A. Improving chemotherapeutic drug penetration in tumors by vascular targeting and barrier alteration. J Clin Invest. 2002;110(4):475-482.

75. Chen Y, Wu JJ, Huang L. Nanoparticles targeted with NGR motif deliver c-myc siRNA and doxorubicin for anticancer therapy. Mol Ther. 2010;18(4):828-834.

76. Pastorino F, Brignole C, Marimpietri D, et al. Vascular damage and antiangiogenic effects of tumor vessel-targeted liposomal chemotherapy. Cancer Res. 2003;63(21):7400-7409. 
77. Matsui M, Shimizu Y, Kodera Y, Kondo E, Ikehara Y, Nakanishi H. Targeted delivery of oligomannose-coated liposome to the omental micrometastasis by peritoneal macrophages from patients with gastric cancer. Cancer Sci. 2010;101(7):1670-1607.

78. Pouvreau I, Zech JC, Thillaye-Goldenberg B, Naud MC, Van ROoijen N, de Kozak Y. Effect of macrophage depletion by liposomes containing dichloromethylene-diphosphonate on endotoxin-induced uveitis. J Neuroimmunol. 1998;86(2):171-181.

79. Killion JJ, Fidler IJ. Therapy of cancer metastasis by tumoricidal activation of tissue macrophages using liposome-encapsulated immunomodulators. Pharmacol Ther. 1998;78(3):141-154.

80. Nardin A, Lefebvre ML, Labroquère K, Faure O, Abastado JP. Liposomal muramyl tripeptide phosphatidylethanolamine: Targeting and activating macrophages for adjuvant treatment of osteosarcoma. Curr Cancer Drug Targets. 2006;6(2):123-133.

81. Coffelt SB, Hughes R, Lewis CE. Tumor-associated macrophages: effectors of angiogenesis and tumor progression. Biochim Biophys Acta. 2009;1796(1):11-18.

82. Martinez FO, Sica A, Mantovani A, Locati M. Macrophage activation and polarization. Front Biosci. 2008;13:453-461.

83. Banciu M, Metselaar JM, Schiffelers RM, Storm G. Antitumor activity of liposomal prednisolone phosphate depends on the presence of functional tumor-associated macrophages in tumor tissue. Neoplasia. 2008;10(2):108-117.

84. Banciu M, Schiffelers RM, Storm G. Investigation into the role of tumor-associated macrophages in the antitumor activity of Doxil. Pharm Res. 2008;25(8):1948-1955.

85. Laakkonen $\mathrm{P}$, Akerman ME, Biliran H, et al. Antitumor activity of a homing peptide that targets tumor lymphatics and tumor cells. Proc Natl Acad Sci U S A. 2004;101(25):9381-9386.

86. Fogal V, Zhang L, Krajewski S, Ruoslahti E. Mitochondrial/cell-surface protein $\mathrm{p} 32 / \mathrm{gC} 1 \mathrm{qR}$ as a molecular target in tumor cells and tumor stroma. Cancer Res. 2008;68(17):7210-7218.

87. Yan Z, Wang F, Wen Z, et al. LyP-1-conjugated PEGylated liposomes: A carrier system for targeted therapy of lymphatic metastatic tumor. J Control Release. 2012;157(1):118-125.

88. Baeten D, Demetter P, Cuvelier CA, et al. Macrophages expressing the scavenger receptor CD163: a link between immune alterations of the gut and synovial inflammation in spondyloarthropathy. J Pathol. 2002; 196(3):343-350.

89. Jensen TO, Schmidt H, Møller HJ, et al. Macrophage markers in serum and tumor have prognostic impact in American Joint Committee on Cancer stage I/II melanoma. J Clin Oncol. 2009;27(20): 3330-3337.
90. Etzerodt A, Maniecki MB, Graversen JH, Møller HJ, Torchilin VP, Moestrup SK. Efficient intracellular drug-targeting of macrophages using stealth liposomes directed to the hemoglobin scavenger receptor CD163. J Control Release. 2012;160(1):72-80.

91. Ikehara Y, Niwa T, Biao L, et al. A carbohydrate recognition-based drug delivery and controlled release system using intraperitoneal macrophages as a cellular vehicle. Cancer Res. 2006;66(17): 8740-8748.

92. Baum P, Müller D, Rüger R, Kontermann RE. Single-chain Fv immunoliposomes for the targeting of fibroblast activation proteinexpressing tumor stromal cells. J Drug Target. 2007;15(6):399-406.

93. Sadlonova A, Bowe DB, Novak Z, et al. Identification of molecular distinctions between normal breast-associated fibroblasts and breast cancer-associated fibroblasts. Cancer Microenviron. 2009;2(1):9-21.

94. Xian X, Håkansson J, Ståhlberg A, et al. Pericytes limit tumor cel metastasis. J Clin Invest. 2006;116(3):642-651.

95. Kitadai Y, Sasaki T, Kuwai T, et al. Expression of activated plateletderived growth factor receptor in stromal cells of human colon carcinomas is associated with metastatic potential. Int J Cancer. 2006;119(11):2567-2574.

96. Prakash J, de Jong E, Post E, Gouw AS, Beljaars L, Poelstra K. A novel approach to deliver anticancer drugs to key cell types in tumors using a PDGF receptor binding cyclic peptide containing carrier. J Control Release. 2010;145(2):91-101.

97. Chiquet-Ehrismann R, Chiquet M. Tenascins: regulation and putative functions during pathological stress. J Pathol. 2003;200(4): 488-499.

98. Shao K, Hou Q, Go ML, et al. Sulfatide-tenascin interaction mediates binding to the extracellular matrix and endocytic uptake of liposomes in glioma cells. Cell Mol Life Sci. 2007;64(4):506-515.

99. Achen MG, Mann GB, Stacker SA. Targeting lymphangiogenesis to prevent tumour metastasis. Br J Cancer. 2006;94(10): 1355-1360.

100. Mandriota SJ, Jussila L, Jeltsch M, et al. Vascular endothelial growth factor-C-mediated lymphangiogenesis promotes tumour metastasis. EMBO J. 2001;20(4):672-682.

101. Wen J, Fu AF, Chen LJ, et al. Liposomal honokiol inhibits VEGF-Dinduced lymphangiogenesis and metastasis in xenograft tumor model. Int J Cancer. 2009;124(11):2709-2718.
International Journal of Nanomedicine

\section{Publish your work in this journal}

The International Journal of Nanomedicine is an international, peerreviewed journal focusing on the application of nanotechnology in diagnostics, therapeutics, and drug delivery systems throughout the biomedical field. This journal is indexed on PubMed Central,

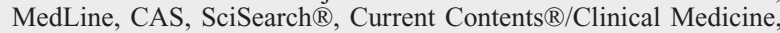

\section{Dovepress}

Journal Citation Reports/Science Edition, EMBase, Scopus and the Elsevier Bibliographic databases. The manuscript management system is completely online and includes a very quick and fair peer-review system, which is all easy to use. Visit http://www.dovepress.com/ testimonials.php to read real quotes from published authors. 